\title{
Comparative Analysis of the Performance of Commonly Available Density Functionals in the Determination of Geometrical Parameters for Zinc Complexes
}

\author{
SÉRGIO F. SOUSA, EMANUELA S. CARVALHO, DIANA M. FERREIRA, ISABEL S. TAVARES, \\ PEDRO A. FERNANDES, MARIA JOÃO RAMOS, JOSÉ A. N. F. GOMES \\ REQUIMTE, Departamento de Química, Faculdade de Cióncias, Universidade do Porto, \\ Rua do Campo Alegre, 687, 4169-007 Porto, Portugal \\ Received 8 January 2009; Accepted 18 March 2009 \\ DOI 10.1002/jcc.21304 \\ Published online 27 April 2009 in Wiley InterScience (www.interscience.wiley.com).
}

\begin{abstract}
A set of 44 Zinc-ligand bond-lengths and of 60 ligand-metal-ligand bond angles from 10 diverse transition-metal complexes, representative of the coordination spheres of typical biological $\mathrm{Zn}$ systems, were used to evaluate the performance of a total of 18 commonly available density functionals in geometry determination. Five different basis sets were considered for each density functional, namely two all-electron basis sets (a double-zeta and triple-zeta formulation) and three basis sets including popular types of effective-core potentials: Los Alamos, Steven-Basch-Krauss, and Stuttgart-Dresden. The results show that there are presently several better alternatives to the popular B3LYP density functional for the determination of $\mathrm{Zn}$-ligand bond-lengths and angles. BB1K, MPWB1K, MPW1K, B97-2 and TPSS are suggested as the strongest alternatives for this effect presently available in most computational chemistry software packages. In addition, the results show that the use of effective-core potentials (in particular Stuttgart-Dresden) has a very limited impact, in terms of accuracy, in the determination of metal-ligand bond-lengths and angles in Zinc-complexes, and is a good and safe alternative to the use of an allelectron basis set such as 6-31G(d) or 6-311G(d,p).
\end{abstract}

(C) 2009 Wiley Periodicals, Inc. J Comput Chem 30: 2752-2763, 2009

Key words: zinc enzymes; metals; DFT; benchmarking; effective-core potentials

\section{Introduction}

Zinc is an essential transition element, necessary for sustaining all life. In fact, Zinc is one of the biologically most abundant and important metal elements (the second most abundant after Iron) and is present in a wide range of enzymes from a broad array of species of all phyla. ${ }^{1-4}$ Zinc is also the only metal known to have representatives in each of the six fundamental classes of enzymes established by the International Union of Biochemistry. ${ }^{2}$ Something like $10 \%$ of the total human proteome (ca. 2800 human proteins) has been proposed to bind Zinc in vivo, ${ }^{5}$ a number, which includes several well-known enzymes such as the carbonic anhydrases I and II, the carboxypeptidases $\mathrm{A}, \mathrm{B}$ and $\mathrm{T}$, alcohol dehydrogenase, and thermolysin. ${ }^{3,6}$ The RNA polymerase II, $^{7-11}$ the matrix metalloproteinases, ${ }^{12-14}$ protein farnesyltransferase, ${ }^{15-20}$ and the metallo- $\beta$-lactamases ${ }^{21,22}$ are among the most discussed examples in recent years.

Beyond this almost ubiquitous presence of Zinc in biology are some of its unusual chemical properties that make this metal very different from the other first-row transition elements, and render it a very appealing target for biological systems. The flexible coordination geometry, the fast ligand exchange, the strong binding to suitable sites, the lack of redox activity (no generation of free radicals), and its role as Lewis acid are just a few examples, which add to a high bioavailability. ${ }^{3,4,23}$ However, the high-biological importance of Zinc and its chemicallyinteresting properties contrast markedly with the relatively limited set of experimental techniques that can be applied to the study of biological Zinc complexes. In fact, the $\mathrm{Zn}$ (II) ion, the only state of this metal present under physiological conditions, is diamagnetic and forms colorless complexes. In addition, no naturally occurring $\mathrm{Zn}$ (II) complexes with (colored) tetrapyrrole ligands exist. For these reasons most spectroscopic techniques normally used in the study of biological metal complexes that

Additional Supporting Information may be found in the online version of this article.

Correspondence to: J.A.N.F. Gomes; e-mail: jfgomes@fc.up.pt

Contract/grant sponsor: FCT (Fundação para a Ciência e a Tecnologia); contract/grant number: POCI/QUI/61563/2004 
explore the UV-Vis (absorption, $\mathrm{CD}$, and MCD) and microwave (EPR, ESEEM, ENDOR, etc.) spectral regions cannot be successfully applied to treat biological Zinc systems, rendering this metal one of the "spectroscopically quiet" metals in biology. ${ }^{24}$ Alternative methodologies play therefore a key role in obtaining insight into the structure of biological Zinc complexes. Synchrotron spectroscopy, X-ray absorption spectroscopy (XAS) and, of course, crystallography are among the few experimental methodologies that can be used with success. For many problems however, the application of theoretical and computational methods is essential to fully understand a given biological Zinc system, both at the structural and mechanistic level. It is therefore not surprising, that the application of theoretical and computational methods in the study of $\mathrm{Zn}$ biological systems has flourished so early in the history of the field. ${ }^{25-28}$

Density functional theory (DFT) is a widely used quantum mechanical methodology with a widespread application in chemistry and physics. With foundations that might be traced back to the 1920 s to the work of Thomas ${ }^{29}$ and Fermi, ${ }^{30}$ DFT methods have emerged as a well-grounded formalism during the 1960s, following the work of Kohn and coworkers ${ }^{31,32}$ in the 1960s. But it was in the 1990s with the development of the B3LYP density functional ${ }^{33-35}$ that the widespread application of DFT has become a reality. DFT offers the advantage of being less computationally demanding than other computational methods that have a similar level of accuracy. In fact, DFT methods are able to include electron correlation in the calculations at a fraction of the time of post-Hartree-Fock methodologies. Today a plethora of density functionals, with different levels of sophistification, accuracy, computational cost and based on very different parameterization philosophies are available, and the number of alternatives continues to grow every year, leading the accuracy of DFT in the determination of chemical properties to new levels. ${ }^{36}$ However, in spite of the wide range of currently available alternatives, most users still continue to rely on the same density functional they did 10 years ago: B3LYP. ${ }^{36}$

The reasons for such a conservative attitude in an otherwise so innovative field of research, as it is this of computational chemistry, reside in great part in the following: B3LYP has marked an age. It was never seen as the perfect density functional, but was extensively used and tested in a wide range of chemical systems and theoretical problems. Its strengths and drawbacks are therefore well known. During more than a decade B3LYP became almost the functional to use "no questions asked." ${ }^{36}$ Given the current standing of the field, a reinterpretation of this paradigm is deeply needed. However, for someone wishing to adopt a new density functional, a great number of very pungent questions immediately arise. No universally accepted choice exists or will probably exist in the near future. The alternatives are extremely diverse and differ on a large variety of aspects (including computational cost). In addition, the performance greatly depends on the problem at hand. During the past few years several studies have tried to evaluate the performance of several density functionals in a variety of aspects, including structure (bonds, angles), ${ }^{37-42}$ kinetics (barrier heights), ${ }^{38,40,41,43-45}$ thermochemistry (atomization energies, binding energies, ionization potentials, electron affinities, heats of formations), ${ }^{37-44,46-48}$ and nonbonded interactions (hydrogen bonding, charge transfer, dipole-interactions, weak interactions, $\pi-\pi$ interactions). ${ }^{38,41,44,49-52}$ However, most of these studies focus exclusively on main group elements. Some efforts in transition metal chemistry have also been reported, but these studies typically rely on small metal dimers, ${ }^{39,40,46,51}$ although some more recent studies incorporating more realistic-sized complexes have also been published. ${ }^{53-56}$

The use of small active-site models treated with DFT-based methodologies is still paramount in the computational study of biological metal systems. ${ }^{57-59}$ For such systems, this type of calculations is at the cornerstone of much of the atomic-level research that is performed, which includes the study of enzyme active-sites and the determination and validation of enzymatic reaction mechanisms, ${ }^{60-64}$ the resolution of mechanistic paradoxes and dilemmas, ${ }^{19,65-67}$ the characterization of metaldependent structural and spectroscopic properties, ${ }^{68-70}$ and the determination of molecular parameters for the application of molecular mechanical methods. ${ }^{71-73}$ Zinc biological complexes represent a particularly challenging subset of such systems, particularly in terms of geometry determination. ${ }^{74}$ The $\mathrm{d}^{10}$ configuration of $\mathrm{Zn}^{2+}$ results in a ligand field-stabilization energy of zero for all possible geometries, leading to spatially not directed (isotropic) polarization effects. On the basis of the electronic structure of the Zinc metal atom, no geometry is intrinsically more stable than another, with the geometry of the complex being mainly determined by the nature and the spatial arrangement of the ligands surrounding the metal atom. Zinc complexes tend therefore to be characterized by particularly flat potential energy surfaces, and by the existence of several low lying energy states connected by low energy barriers, features that make the computational determination of the corresponding geometries particularly tricky and highly-dependent on the combination of method/basis set used. As computational methods are many times the only available tools to gain insight into the structure and mechanism of $\mathrm{Zn}$ biological systems, clearly understanding their performance in the determination of geometries is of great importance.

In this study, instead of restricting our analysis to the study of a small number of ideal metal complexes with bonds, angles (in terms of type and values), dimensions and chemical nature very different from those that would be present in a chemical or biological system of interest, we have tried to focus our analysis on more reasonable systems, with properties closer to those of a system that could realistically be at the center of a given chemical or biological research project. Following this strategy we aim, with this benchmarking study, to bring closer the computational study of Zn systems, to the recent developments in DFT, contributing to a demystification of old ingrained habits and to an increased use of new and improved density functionals in the study of specific chemical problems. So, instead of considering a very small dataset of very simple and highly-symmetric metal complexes with $2-10$ atoms and with geometries determined by high-precision spectroscopy methods or with very-high-level computational methods as it is customary in these studies, we chose to consider an enlarged dataset of Zinc complexes with geometries taken from the Cambridge Structural Database, ${ }^{75}$ with sizes ranging from 15 to 25 atoms, mainly tetrahedral and for which $\mathrm{N}, \mathrm{O}$, and $\mathrm{S}$ coordination to $\mathrm{Zn}$ was predominant as these 
Table 1. Summary of the Density Functionals Considered in this Study.

\begin{tabular}{|c|c|c|c|c|c|c|}
\hline Functionals & Year & Type & $\chi$ & Exchange functional & Correlation functional & Ref. \\
\hline B3LYP & 1994 & H-GGA & 20 & Becke88 & Lee-Yang-Parr & $33-35$ \\
\hline B3P86 & 1993 & H-GGA & 20 & Becke88 & Perdew86 & $33,34,88$ \\
\hline B3PW91 & 1993 & H-GGA & 20 & Becke88 & Perdew-Wang91 & $33,34,89$ \\
\hline B97-2 & 2001 & H-GGA & 21 & B97-2 & B97-2 & 90 \\
\hline $\mathrm{BB} 1 \mathrm{~K}$ & 2004 & HM-GGA & 42 & Becke88 & Becke95 & $33,34,91$ \\
\hline BB95 & 1996 & M-GGA & 0 & Becke88 & Becke95 & 33,34 \\
\hline BLYP & 1988 & GGA & 0 & Becke88 & Lee-Yang-Parr & 33,35 \\
\hline BP86 & 1988 & GGA & 0 & Becke88 & Perdew86 & 33,88 \\
\hline G96LYP & 1996 & GGA & 0 & Gill96 & Lee-Yang-Parr & 35,92 \\
\hline HCTH & 1998 & GGA & 0 & Hamprecht-Cohen-Tozer-Handy & Hamprecht-Cohen-Tozer-Handy & 90 \\
\hline MPW1K & 2000 & H-GGA & 42.8 & modified Perdew-Wang91 & Perdew-Wang91 & $88,89,93,94$ \\
\hline MPW3LYP & 2004 & H-GGA & 21.8 & modified Perdew-Wang91 & Lee-Yang-Parr & $35,89,93$ \\
\hline MPWB1K & 2004 & HM-GGA & 44 & modified Perdew-Wang91 & Becke95 & $34,89,93,95$ \\
\hline PBE & 1996 & GGA & 0 & Perdew-Burke-Ernzerhof & Perdew-Burke-Ernzerhof & 96 \\
\hline SVWN3 & 1981 & LSDA & 0 & Slater & VWN no. 3 & 97,98 \\
\hline TPSS & 2003 & M-GGA & 0 & Tao-Perdew-Staroverov-Scuseria & Tao-Perdew-Staroverov-Scuseria & 99 \\
\hline TPSSh & 2003 & HM-GGA & 10 & Tao-Perdew-Staroverov-Scuseria & Tao-Perdew-Staroverov-Scuseria & 99 \\
\hline VSXC & 1998 & M-GGA & 0 & Van Voorhis-Scuseria & Van Voorhis-Scuseria & 100 \\
\hline
\end{tabular}

$\mathrm{HM}$, Hybrid meta; $\mathrm{H}$, hybrid; $\chi$, percentage of $\mathrm{HF}$ exchange in the functional.

are the most common features that characterize the coordination sphere of Zn biological systems, as recently reviewed. ${ }^{76-78}$

In this study, we evaluated the performance of a total of 18 different commonly available DFT functionals with five different basis sets in the determination of geometrical properties for Zinc complexes. The basis sets considered include two all-electron basis sets (a double-zeta and triple-zeta formulation) and three basis sets involving popular types of effective-core potentials: Los Alamos, Steven-Basch-Krauss, and Stuttgart-Dresden. Special care was taken to ensure a well-balanced selection of commonly available density functionals, i.e. alternatives that have already been made available in the most widely used software packages. ${ }^{36}$ However, the reader should be aware that several other very promising density functionals have been published meanwhile, ${ }^{79-86}$ and are likely to be made available in a near future.

\section{Computational Methods}

Calculations were carried out using the Gaussian 03 suite of programs. ${ }^{87} 18$ different commonly available DFT functionals were tested. The choice of the density functionals used was based in a recent review on the subject, ${ }^{36}$ which presented an overview of the current status of the field and summarized a comprehensive number of benchmarking studies performed over the past 5 years. For this study, particular care was taken to ensure a diverse and representative choice of density functionals. Given the size of the field, we chose to limit this selection to only commonly available density functionals, i.e. alternatives already made available to the general user in the most popular software packages. The list of density functionals considered is presented in Table 1.

A total of five different basis sets were assessed in this study. These are listed in Table 2, together with the valence basis set considered for each of the atom types that make up the Zinc complexes considered. Although in DFT the addition of diffuse functions to a double- $\zeta$ basis set has been shown to be potentially more important than its increase to a triple- $\zeta$ basis set when calculating reaction energies, barrier heights and conformational energies, ${ }^{101}$ in this study the use of such functions for geometry optimization was not attempted. In addition to the two very popular all-electron basis sets - 6-31G(d) and 6-311G(d,p) this study was extended to include three basis sets that use effective core potentials (ECPs). The use of ECPs is grounded

Table 2. Description of the 5 Basis Set Considered in this Study.

\begin{tabular}{|c|c|c|c|c|c|c|c|c|}
\hline \multirow[b]{2}{*}{ Basis set } & \multirow[b]{2}{*}{ Basis set type } & \multicolumn{3}{|c|}{ Valence electrons (if ECPs used) } & \multicolumn{4}{|c|}{ Valence basis set } \\
\hline & & $\mathrm{Zn}$ & $\mathrm{S}, \mathrm{Cl}$ & $\mathrm{C}, \mathrm{N}, \mathrm{O}$ & $\mathrm{Zn}$ & $\mathrm{S}, \mathrm{Cl}$ & $\mathrm{C}, \mathrm{N}, \mathrm{O}$ & $\mathrm{H}$ \\
\hline $6-31 G(d)$ & All Electron & - & - & - & $6 / 6631 / 31 / 1$ & $6 / 631 / 1$ & $6 / 31 / 1$ & 31 \\
\hline $6-311 \mathrm{G}(\mathrm{d}, \mathrm{p})$ & All Electron & - & - & - & $6 / 6631 / 31 / 1$ & $631111 / 42111 / 1$ & $6 / 6311 / 1$ & $311 / 1$ \\
\hline CEP-121G & Small Core & 20 & 6,7 & $4,5,6$ & $4211 / 411$ & 121 & 121 & 311 \\
\hline LanL2DZ & Large Core & 12 & 6,7 & - & $21 / 11 / 41$ & $21 / 21$ & $721 / 41$ & 31 \\
\hline SDD & Small Core & 20 & - & - & $311111 / 22111 / 411$ & $531111 / 4211$ & $6111 / 41$ & 31 \\
\hline
\end{tabular}


on the idea that the chemically inert core electrons, whose description typically requires a large set of gaussians, can be substituted by an approximate function, with large savings in terms of computational cost and moderate penalty in terms of accuracy. The approximate function used is called pseudopotential or effective core potential. In practice, the use of such functions significantly reduces the number of electrons that are explicitly described in the calculation, thereby lowering the computational expense associated. As many chemical properties such as bond strengths, electron affinities, polarizabilities, ionization potentials, as well as molecular geometries, are essentially determined by the valence electrons, the use of ECPs is generally a very reasonable choice when properties related to the valence electron system are to be investigated. Although the idea behind the use of ECPs is not new, their use has become increasingly popular during the past years, particularly in the description of systems that include metal atoms.

In this study three different types of ECPs were considered. The basis set CEP-121G uses the Steven-Basch-Krauss pseudopotentials ${ }^{102-104}$ (sometimes credited as SKBJ), LanL2DZ uses the Los Alamos pseudopotentials (sometimes referred as Hay and Wadt), ${ }^{105-107}$ whereas SDD uses the Stuttgart-Dresden pseudopotentials $^{108,109}$ (also known as Stoll-Preuss, or simply SP). Details concerning these pseudopotentials, and the corresponding schemes included in Gaussian 03 for the treatment of $\mathrm{Zn}$ and of the directly coordinating atoms are described in Table 2, together with a comparison with the two all-electron basis sets considered in this study.

The dataset used to evaluate the performance of the 90 combinations density functional/basis set tested comprised a total of $44 \mathrm{Zn}$-Ligand bond-lengths (including $15 \mathrm{Zn}-\mathrm{O}$ bonds, $17 \mathrm{Zn}-\mathrm{N}$ bonds, $8 \mathrm{Zn}-\mathrm{S}$ bonds, and $4 \mathrm{Zn}-\mathrm{Cl}$ bonds) and 60 Ligand-ZnLigand Angles obtained from 10 structurally diverse transitionmetal complexes. These are presented in Figure 1 with details given in Table 3. In this work, instead of trying to focus our analysis on small $\mathrm{Zn}$ molecules, which typically present characteristics that are very different from those of a biological Zinccomplex of interest, we aimed to evaluate the performance of the several density functionals and basis sets on the determination of bond-lengths and angles in a more realistic setting, i.e. in

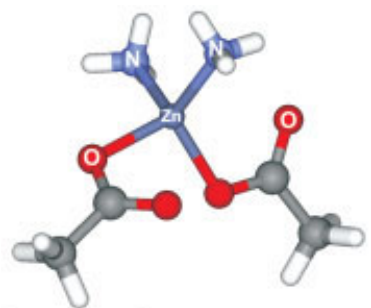

Complex 1

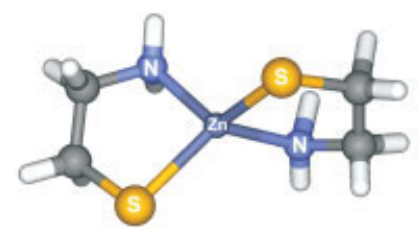

Complex 2

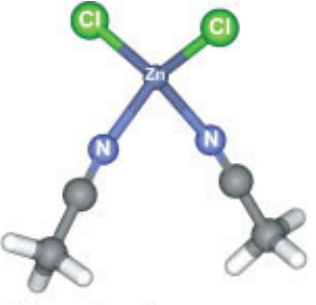

Complex 3

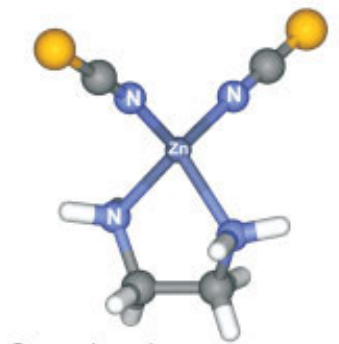

Complex 4

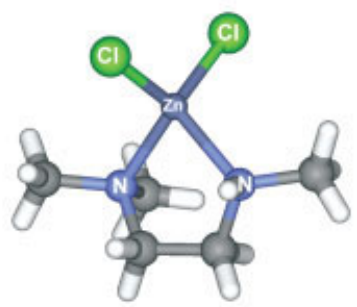

Complex 7

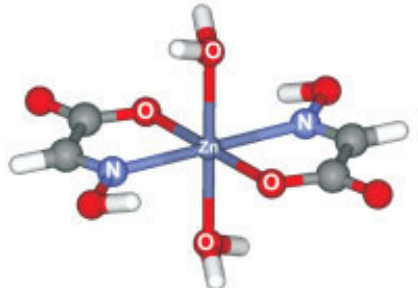

Complex 5

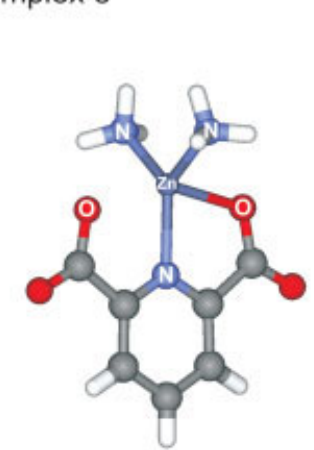

Complex 8
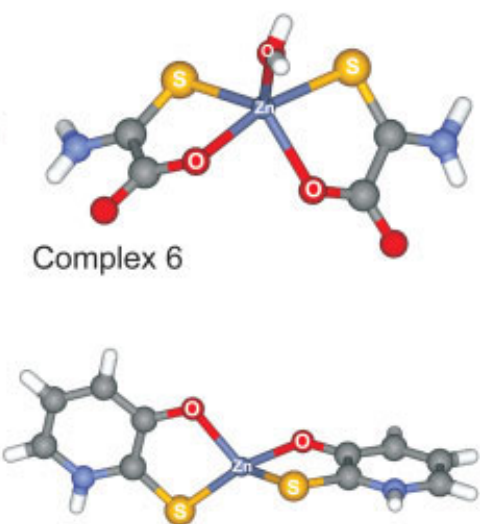

Complex 9

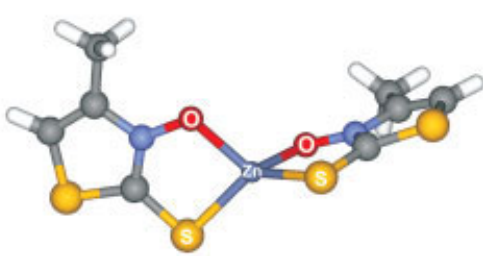

Complex 10

Figure 1. Structure and topology of the zinc complexes considered in this study. 
Table 3. Summary of the Zinc Complexes Considered in this Study.

\begin{tabular}{lllcrr}
\hline Structure & CSD code & Coordination sphere & No. atoms & R-factor & Reference \\
\hline Complex 1 & PVVAUM01 & N-N-O-O & 23 & 0.028 & 110 \\
Complex 2 & XUSTAG & N-N-S-S & 21 & 0.033 & 75 \\
Complex 3 & ZNACET01 & N-N-Cl-Cl & 15 & 0.027 & 111 \\
Complex 4 & GOVLAE & N-N-N-N & 19 & 0.050 & 112 \\
Complex 5 & FIDWOF & N-N-O-O-O-O & 23 & 0.031 & 113 \\
Complex 6 & GAYZIP & O-O-O-S-S & 20 & 0.023 & 114 \\
Complex 7 & BEVHAM & N-N-Cl-Cl & 24 & 0.040 & 115 \\
Complex 8 & ECUQAV & N-N-N-O-O & 24 & 0.028 & 116 \\
Complex 9 & OKEZOT & O-O-S-S & 25 & 0.040 & 75 \\
Complex 10 & UCUZIB & O-O-S-S & 25 & 0.031 & 117 \\
\hline
\end{tabular}

treating reasonable-sized systems, with dimensions and characteristics closer to those of a biological Zinc-complex of interest. However, for such large systems high-precision spectroscopy data is unavailable, and the application of very-high level computational methodologies, such as coupled cluster, configuration interaction or high-order Moller-Plesset theory to optimize geometries is still too computationally intensive for practical use. Structures for the 10 Zinc-complexes considered in this study, were therefore taken from the Cambridge Structural Database, ${ }^{75}$ which presently holds more than 400,000 crystallographic structures of small molecules, with almost 250,000 of these being of transition metal compounds. Particular care was taken in selecting the 10 Zinc-complexes used as reference, with the choice being made according to the following aspects: (1) Low r-factor (below 0.050); (2) Reasonable dimension, i.e. structures with a number of atoms consistent with those of a typically studied biological Zinc-complex (higher that 15 atoms), but small enough to allow the efficient application of the several combinations method/basis set evaluated (less than 30 atoms); (3) Prevalence for $\mathrm{Zn}-\mathrm{O}, \mathrm{Zn}-\mathrm{N}$, and $\mathrm{Zn}-\mathrm{S}$ coordination, as these are the most common interactions in Zinc-biological complexes; ${ }^{76-78}$ (4) Preference for a coordination number of 4 and for a distorted tetrahedral geometry around the metal atom, as it is characteristic of Zinc-biological complexes; ${ }^{76-78}$ (5) Diversity in terms of the groups present at the metal coordination sphere. The structures for these complexes are presented in Supporting Information.

Each of these structures was energy minimized using the 18 density functionals described in Table 1, together with each one of the five basis sets presented in Table 2. Geometries were freely optimized individually for each combination density functional/basis set, starting from the initial CSD structure. The metal-ligand bond-lengths and angles in the resulting structures were then measured and compared with the initial CSD values. The reader should however be aware that comparing equilibrium structures in the gas-phase with thermally averaged crystallographic structures is by itself an approximation, and has a certain degree of error associated. Although this type of comparison is a standard practice in computational chemistry, some systematic differences between both types of geometries have been described in the literature. ${ }^{118}$ In particular, gas-phase geometries are typically more symmetric, and have been found to have slightly increased metalligand bonds-lengths, while the bond-angles do not generally change significantly apart from the natural adjustment that arises from the symmetrization of the structure. ${ }^{118} \mathrm{~A}$ minimum baseline uncertainty in metal-ligand bond-lengths of ca. $0.01 \AA$ and in valence angles of ca. $1^{\circ}-2^{\circ}$ has been attributed to the effect of the crystal environment on metal structures. ${ }^{119-121}$

\section{Results and Discussion}

Table 4 presents the mean signed errors (MSEs) calculated for each one of the 90 combinations of method/basis set tested, considering all the Zn-ligand bond-lengths in the set of $10 \mathrm{Zinc}$ complexes considered in this study. The MSE is taken as the difference between the values calculated with the combination of method/basis set and the values reported in the initial CSD structures. The results show that the Zn-ligand bond-lengths are typically overestimated (average MSE $0.033 \AA$ for all the 90 combinations). Considering the five basis sets evaluated MPW1K gives the lowest MSE, closely followed by MPWB1K, SVWN3, and BB1K (average MSE values of 0.006, 0.009, 0.012 , and $0.015 \AA$, respectively), whereas HCTH, BLYP, and G96LYP exhibit the largest MSEs $(0.059,0.055$, and $0.052 \AA)$. Four out of the five basis sets tested display average MSEs lower than $0.031 \AA$, the exception being the LanL2DZ basis set (MSE of $0.067 \AA$ ).

Table 5 presents the corresponding mean unsigned errors (MUEs), taken as the module of the difference between the values calculated with the combination of method/basis set and the values reported in the initial CSD structures. Globally, the results indicate an average MUE of $0.084 \AA$ in the determination of $\mathrm{Zn}$-ligand bond-lengths for the 18 density functionals and 5 basis sets considered. The average MUE obtained for this test set of $10 \mathrm{Zn}$ complexes is almost one order of magnitude higher than the average MUEs calculated in a recent study that considered 10 small $\mathrm{Zn}$ model compounds with reference structures determined at the $\operatorname{CCSD}(\mathrm{T})$ level, and which employed higher quality basis sets. ${ }^{56}$

In the present study, the results show a large improvement in terms of MUE in the determination of Zn-ligand bond-lengths when moving from the LSDA density functionals (SVWN3) to the GGA and M-GGA alternatives, a feature not easily noticed by looking simply to the MSE values on Table 4 . The perform- 
Table 4. Calculated Mean Signed Error (MSE) in the Zn-Ligand Bond-Lengths ( $\mathrm{A}$ ).

\begin{tabular}{llrrrrrr}
\hline Type & Functional & $6-31 \mathrm{G}(\mathrm{d})$ & $6-311 \mathrm{G}(\mathrm{d}, \mathrm{p})$ & CEP-121G & LanL2DZ & SDD & Av \\
\hline LSDA & SVWN3 & -0.031 & -0.017 & 0.053 & 0.052 & 0.000 & 0.012 \\
GGA & BLYP & 0.042 & 0.056 & 0.027 & 0.091 & 0.057 & 0.055 \\
& BP86 & 0.031 & 0.016 & 0.103 & 0.068 & 0.031 & 0.050 \\
& G96LYP & 0.024 & 0.041 & 0.059 & 0.087 & 0.050 & 0.052 \\
& HCTH & 0.033 & 0.049 & 0.063 & 0.092 & 0.058 & 0.059 \\
& PBE & 0.028 & 0.059 & 0.042 & 0.068 & 0.029 & 0.045 \\
M-GGA & BB95 & 0.039 & 0.042 & 0.038 & 0.092 & 0.033 & 0.049 \\
& TPSS & -0.002 & 0.018 & 0.004 & 0.061 & 0.027 & 0.022 \\
& VSXC & 0.034 & 0.046 & 0.042 & 0.087 & 0.032 & 0.048 \\
H-GGA & B3LYP & 0.044 & 0.026 & 0.020 & 0.066 & 0.021 & 0.035 \\
& B3P86 & 0.020 & 0.019 & 0.000 & 0.047 & 0.036 & 0.025 \\
& B3PW91 & 0.000 & 0.028 & 0.047 & 0.054 & 0.012 & 0.028 \\
& B97-2 & 0.015 & 0.024 & 0.024 & 0.061 & 0.021 & 0.029 \\
& MPW1K & 0.007 & -0.003 & -0.004 & 0.034 & -0.005 & 0.006 \\
& MPW3LYP & 0.039 & 0.024 & 0.018 & 0.064 & 0.022 & 0.033 \\
HM-GGA & BB1K & -0.005 & 0.005 & 0.012 & 0.064 & -0.002 & 0.015 \\
& MPWB1K & -0.011 & -0.002 & 0.006 & 0.058 & -0.005 & 0.009 \\
\multirow{2}{*}{ Average } & TPSSh & 0.024 & 0.015 & 0.011 & 0.055 & 0.014 & 0.024 \\
& & 0.018 & 0.025 & 0.031 & 0.067 & 0.024 & 0.033 \\
\hline
\end{tabular}

ance of the H-GGAs and of the HM-GGAs was in general at the same level to that of the M-GGAs. Among the GGAs, G96LYP gave the lowest MUE $(0.083 \AA)$, whereas in the M-GGA family the TPSS density functional gave the best results (average MUE of $0.072 \AA$ ), and was ranked third in the test among the 18 density functionals evaluated in the determination of $\mathrm{Zn}$-ligand bond-lengths. MPW1K and B97-2 gave the best results among the H-GGAs with average MUEs of 0.073 and $0.076 \AA$, whereas BB1K and MPWB1K (average MUEs of $0.071 \AA$ ) were first among the HM-GGAs, and gave the lowest average MUEs values from the 18 density functionals considered in the study.
In terms of basis sets, the results show that the use of the triple-zeta basis set 6-311G(d,p) offers a very small advantage in terms of accuracy in comparison with the use of the double-zeta basis set $6-31 \mathrm{G}(\mathrm{d})$, with average MUEs of 0.079 and $0.076 \AA$, respectively. Table 5 also reveals that the magnitude of the difference in accuracy between these two basis sets depends on the density functional considered, with the more sophisticated $\mathrm{H}$ GGAs and HM-GGAs showing larger differences in the MUE values calculated with these two basis sets. In the particular case of B3LYP and MPW3LYP, this improvement in accuracy in the determination of $\mathrm{Zn}$-ligand bond-lengths, in terms of MUE, is

Table 5. Calculated Mean Unsigned Error (MUE) in the Zn-Ligand Bond-Lengths ( $\mathrm{A}$ ).

\begin{tabular}{lllccccc}
\hline Type & Functional & $6-31 \mathrm{G}(\mathrm{d})$ & $6-311 \mathrm{G}(\mathrm{d}, \mathrm{p})$ & CEP-121G & LanL2DZ & SDD & Av \\
\hline LSDA & SVWN3 & 0.101 & 0.095 & 0.168 & 0.105 & 0.114 & 0.117 \\
GGA & BLYP & 0.084 & 0.088 & 0.077 & 0.120 & 0.082 & 0.090 \\
& BP86 & 0.088 & 0.069 & 0.165 & 0.107 & 0.061 & 0.098 \\
& G96LYP & 0.068 & 0.081 & 0.077 & 0.116 & 0.074 & 0.083 \\
& HCTH & 0.077 & 0.084 & 0.084 & 0.120 & 0.083 & 0.090 \\
& PBE & 0.089 & 0.096 & 0.074 & 0.103 & 0.059 & 0.084 \\
M-GGA & BB95 & 0.092 & 0.084 & 0.064 & 0.110 & 0.063 & 0.083 \\
& TPSS & 0.055 & 0.064 & 0.079 & 0.100 & 0.060 & 0.072 \\
& VSXC & 0.073 & 0.083 & 0.079 & 0.108 & 0.072 & 0.083 \\
H-GGA & B3LYP & 0.098 & 0.071 & 0.077 & 0.103 & 0.078 & 0.085 \\
& B3P86 & 0.088 & 0.078 & 0.066 & 0.091 & 0.093 & 0.083 \\
& B3PW91 & 0.059 & 0.080 & 0.118 & 0.094 & 0.068 & 0.084 \\
& B97-2 & 0.066 & 0.070 & 0.072 & 0.099 & 0.073 & 0.076 \\
& MPW1K & 0.075 & 0.063 & 0.072 & 0.088 & 0.065 & 0.073 \\
& MPW3LYP & 0.099 & 0.070 & 0.076 & 0.101 & 0.074 & 0.084 \\
HM-GGA & BB1K & 0.059 & 0.063 & 0.056 & 0.112 & 0.063 & 0.071 \\
& MPWB1K & 0.061 & 0.065 & 0.055 & 0.115 & 0.061 & 0.071 \\
& TPSSh & 0.089 & 0.070 & 0.078 & 0.099 & 0.066 & 0.080 \\
Average & & 0.079 & 0.076 & 0.093 & 0.105 & 0.073 & 0.084 \\
\hline
\end{tabular}


Table 6. Relative CPU Timings for the 5 Basis Sets Considered in this Study and for 5 Representative Density Functionals as Calculated for Complex 10, Giving an Indication of the General Magnitude of the Differences, In Terms of Computational Cost, Between the Several Alternatives (CPU Timing for B3LYP/6-31G(d) Taken as Reference).

\begin{tabular}{llcccccc}
\hline Type & Functional & 6-31G(d) & 6-311G(d,p) & CEP-121G & LanL2DZ & SDD & Average \\
\hline LSDA & SVWN3 & 0.5 & 1.1 & 0.3 & 0.3 & 0.3 & 0.6 \\
GGA & G96LYP & 0.9 & 1.1 & 0.6 & 0.4 & 0.5 & 0.9 \\
M-GGA & TPSS & 1.2 & 2.5 & 1.6 & 0.6 & 1.1 & 1.7 \\
H-GGA & B3LYP & 1.0 & 1.7 & 0.4 & 0.4 & 0.5 & 1.0 \\
HM-GGA & BB1K & 1.5 & 1.6 & 0.9 & 0.6 & 1.0 & 1.4 \\
Average & & 1.0 & 1.6 & 0.7 & 0.5 & 0.7 & \\
\hline
\end{tabular}

particularly high and amounts to 0.027 and $0.029 \AA$, respectively. Globally however, taking into consideration that the computational cost of 6-311G(d,p) is much higher than the one associated with a $6-31 \mathrm{G}(\mathrm{d})$ description, the results demonstrate that the gain in terms of accuracy in the determination of Zn-ligand bond-lengths is in general not significant enough to justify the use of a $6-311 \mathrm{G}(\mathrm{d}, \mathrm{p})$ over that of a $6-31 \mathrm{G}(\mathrm{d})$ basis set. Table 6 gives an indication of the relative timings (in terms of computational cost) associated to the different basis sets considered in this study for five different and representative density functionals (one from each family). A detailed comparison of the computational time required in geometry optimization processes for different density functionals and basis sets is a difficult task, which depends on several different aspects. Examples include the number of basis functions associated, the time required by SCF cycle, the number of SCF cycles required for SCF convergence, and the number of geometries required for that a final optimized geometry can be obtained. The relative timings (reference B3LYP/6-31G(d)) presented in Table 6 and determined for complex 10 are merely illustrative of the global trends normally encountered, and should be seen as a first and rough guideline. The reader is advised however that any of the above mentioned aspects, which depend from molecule to molecule, may lead to significant alterations on these relative values.

The performance of the basis sets that include ECPs were the subject of particular interest in this study, as their use in the treatment of systems that include metal atoms is much less computationally demanding than the one associated to the use of the all-electron basis sets, such as the ones described earlier (Table 6). The results presented in Table 5 show that these three basis sets performed rather well in the determination of Zn-ligand bond-lengths with an average MUE of less than $0.1 \AA$ for the majority of the density functionals tested. The SDD basis set, which incorporates the Stuttgart-Dresden ECPs gave the best results, with an average MUE of $0.073 \AA$, a value even better than the one obtained with the all-electron basis set 6$311 \mathrm{G}(\mathrm{d}, \mathrm{p})$, and only at a fraction of the computational cost associated (Table 6). CEP-121G Steven-(Basch-Krauss pseudopotentials) displayed also a rather good accuracy in the calculation of Zn-ligand bond-lengths, with an average MUE of only $0.093 \AA$. The low computational cost associated to the ECP basis set and the relatively good performance demonstrated render this basis sets a competitive alternative to the all-electron formulations tested. LanL2DZ (Los Alamos pseudopotential), with a MUE $0.105 \AA$, exhibited a relatively poor performance in comparison with the other two basis sets that include ECPs.

Recently, a new and more flexible basis set that employs the Los Alamos pseudopotential has been made available in the EMSL Basis Set Exchange. ${ }^{122}$ To check if the apparent deficiencies of the LanL2DZ basis set were associated to the small valence basis set that come with this ECP, the performance of the much more flexible LanL2TZ basis set was investigated for one of the complexes studied (complex 10). This new basis set, denoted LanL2TZ, involves the use of a new contraction scheme for $\mathrm{Zn}[5 \mathrm{~s}, 5 \mathrm{p}, 3 \mathrm{~d}]$, whereas the main group atoms with an ECP (like sulphur) are described by a fully uncontracted formulation. Five different density functionals were considered in this evaluation. Results are presented in Table 7, illustrating also the per-

Table 7. Comparison of the Performance of the New LanL2TZ Basis Set With the Other Basis Sets that Include ECPs for Complex 10 Considering 5 Representative Density Functionals.

\begin{tabular}{|c|c|c|c|c|c|}
\hline \multirow{2}{*}{$\begin{array}{l}\text { Density } \\
\text { functional }\end{array}$} & \multirow[b]{2}{*}{ Basis set } & \multicolumn{2}{|c|}{ Bond-lengths ( } & \multicolumn{2}{|c|}{ Angles $\left({ }^{\circ}\right)$} \\
\hline & & MSE & MEU & MSE & MEL \\
\hline \multirow[t]{4}{*}{ SVWN3 } & CEP-121G & 0.001 & 0.036 & 0.0 & 2.7 \\
\hline & LanL2DZ & 0.062 & 0.087 & 0.3 & 4.3 \\
\hline & LanL2TZ & 0.065 & 0.085 & 0.3 & 4.0 \\
\hline & SDD & -0.006 & 0.044 & 0.1 & 2.1 \\
\hline \multirow[t]{4}{*}{ G96LYP } & CEP-121G & 0.073 & 0.073 & 0.1 & 2.4 \\
\hline & LanL2DZ & 0.128 & 0.128 & 0.4 & 4.8 \\
\hline & LanL2TZ & 0.132 & 0.132 & 0.3 & 4.6 \\
\hline & SDD & 0.072 & 0.072 & 0.1 & 2.6 \\
\hline \multirow[t]{4}{*}{ TPSS } & CEP-121G & 0.054 & 0.054 & 0.1 & 2.8 \\
\hline & LanL2DZ & 0.108 & 0.108 & 0.3 & 5.0 \\
\hline & LanL2TZ & 0.112 & 0.112 & 0.3 & 4.7 \\
\hline & SDD & 0.053 & 0.055 & 0.1 & 2.7 \\
\hline \multirow[t]{4}{*}{ B3LYP } & CEP-121G & 0.057 & 0.057 & 0.2 & 2.6 \\
\hline & LanL2DZ & 0.106 & 0.106 & 0.4 & 5.0 \\
\hline & LanL2TZ & 0.109 & 0.109 & 0.4 & 4.9 \\
\hline & SDD & 0.054 & 0.061 & 0.2 & 3.1 \\
\hline \multirow{4}{*}{ BB1K } & CEP-121G & 0.028 & 0.051 & 0.2 & 3.0 \\
\hline & LanL2DZ & 0.071 & 0.090 & 0.4 & 5.6 \\
\hline & LanL2TZ & 0.075 & 0.090 & 0.4 & 5.5 \\
\hline & SDD & 0.025 & 0.057 & 0.2 & 3.9 \\
\hline
\end{tabular}


formance, for this individual complex, of the other basis sets that use ECPs considered in this study. Globally, the results show that the performance of this new LanL2TZ basis set in the determination of $\mathrm{Zn}$ bond-lengths and angles is at the same level of the LanL2DZ basis set, and is significantly poorer than the SDD and CEP-121G alternatives. This observation suggests that the relatively poor performance of the LanL2DZ basis set is not a result of the basis set used with the atoms for which the Los Alamos pseudopotential is applied. This result could be due to a deficiency in the Los Alamos ECP for Zinc, or most likely, the basis set considered for the elements in the first and second period (in complex 10, hydrogen, carbon, nitrogen, and oxygen). For these elements with both the LanL2DZ and LanL2TZ basis sets, an all-electron double zeta D95V basis set is employed.

Looking at the full set of 90 combinations of method/basis set tested, the best results for the determination of Zn-ligand bond-lengths were obtained with TPSS/6-31G(d) and MPWB1K/ CEP-121G (average MUE of $0.055 \AA$ ), BB1K/CEP-121G (average MUE of $0.056 \AA$ ), and $\mathrm{BB} 1 \mathrm{~K} / 6-31 \mathrm{G}(\mathrm{d}), \mathrm{PBE} / \mathrm{SDD}$, and B3PW91/6-31G(d) (average MUE of $0.059 \AA$ ). Particularly poor combinations were those of SVWN3 and BP86 with the CEP$121 \mathrm{G}$ basis set (average MUEs of 0.168 and $0.165 \AA$ ) and of BLYP with LanL2DZ (average MUE of $0.120 \AA$ ).

To further complement this analysis on the performance of 18 commonly available density functionals and 5 basis sets for the determination of Zn-ligand bond-lengths, MUE values were calculated for different $\mathrm{Zn}$-ligand bond types. Results are presented in Table 8. Globally, the results show that the average MUE is generically the same for $\mathrm{Zn}-\mathrm{O}$ and $\mathrm{Zn}-\mathrm{N}$ bonds, with values of 0.087 and $0.083 \AA$, respectively. For $\mathrm{Zn}-\mathrm{S}$ bonds this value is significantly higher $(0.102 \AA)$, whereas for $\mathrm{Zn}-\mathrm{Cl}$ an av- erage MUE of only $0.041 \AA$ was obtained, although only 4 Zn$\mathrm{Cl}$ bonds were present in the dataset considered. In general, small differences in the performance of the several density functionals were encountered for different bond-types. For Zn-O coordination, the density functionals TPSS, G96LYP, and BLYP provided the best results (average MUE values between 0.063 and $0.069 \AA$ ), with SVWN3 and BP86 giving the highest average MUEs (0.190 and $0.105 \AA$, respectively). In $\mathrm{Zn}-\mathrm{N}$ bonds, the best results were obtained with the MPWB1K, BB1K, and MPW1K density functionals (average MUE 0.059, 0.061, and $0.064 \AA$, respectively). B3PW91 gave the worst results for this type of interaction with an average MUE of $0.132 \AA$. In the treatment of $\mathrm{Zn}-\mathrm{S}$ interactions the lowest average MUE error was obtained surprisingly with the SVWN3 density functional (average MUE $0.058 \AA$ ), with MPWB1K, BB1K and MPW1K once again on the top positions (average MUE of $0.075,0.082$, and $0.082 \AA$, respectively). BLYP, G96LYP and HCTH gave the worst results for $\mathrm{Zn}-\mathrm{S}$ bonds with average MUE higher than $0.121 \AA$. Finally, for the description of $\mathrm{Zn}-\mathrm{Cl}$ bonds the best results were obtained with the density functionals MPW1K, BB1K, and MPWB1K with average MUEs of 0.028, 0.029, and $0.029 \AA$, respectively, whereas the five GGA density functionals tested, with average MUEs higher than $0.052 \AA$, were the worst in the test.

Table 9 presents the average MUE calculated for each bond type arranged by basis set used. The results show that for $\mathrm{Zn}-\mathrm{O}$ bond-lengths the average MUE decreases from $0.123 \AA$ with 6$31 \mathrm{G}(\mathrm{d})$ to $0.079 \AA$ with $6-311 \mathrm{G}(\mathrm{d}, \mathrm{p})$, with the use of basis sets containing ECPs resulting in even lower average MUE values. For $\mathrm{Zn}-\mathrm{N}$ interactions, however, the best performance was obtained with the 6-31G(d) basis set (average MUE of $0.061 \AA$ ).

Table 8. Calculated Mean Unsigned Error (MUE) in the Zn-Ligand Bond-Lengths ( $\mathrm{A}$ ) by Type of Coordination.

\begin{tabular}{lllllll}
\hline Type & Functional & $\begin{array}{c}\mathrm{Zn}-\mathrm{O} \\
(15 \text { bonds })\end{array}$ & $\begin{array}{c}\mathrm{Zn}-\mathrm{N} \\
(17 \text { bonds })\end{array}$ & $\begin{array}{c}\mathrm{Zn}-\mathrm{S} \\
(8 \text { bonds })\end{array}$ & $\begin{array}{c}\mathrm{Zn}-\mathrm{Cl} \\
(4 \text { bonds })\end{array}$ & $\begin{array}{c}\mathrm{Zn}-\text { All } \\
(15 \text { bonds })\end{array}$ \\
\hline LSDA & SVWN3 & 0.190 & 0.088 & 0.058 & 0.042 & 0.117 \\
GGA & BLYP & 0.069 & 0.096 & 0.135 & 0.060 & 0.090 \\
& BP86 & 0.105 & 0.086 & 0.106 & 0.052 & 0.098 \\
& G96LYP & 0.067 & 0.085 & 0.128 & 0.057 & 0.083 \\
& HCTH & 0.075 & 0.103 & 0.121 & 0.050 & 0.090 \\
M-GGA & PBE & 0.077 & 0.095 & 0.101 & 0.048 & 0.084 \\
& BB95 & 0.080 & 0.085 & 0.103 & 0.045 & 0.083 \\
& TPSS & 0.063 & 0.077 & 0.108 & 0.043 & 0.072 \\
H-GGA & VSXC & 0.075 & 0.104 & 0.099 & 0.037 & 0.083 \\
& B3LYP & 0.089 & 0.068 & 0.118 & 0.042 & 0.085 \\
& B3P86 & 0.093 & 0.076 & 0.096 & 0.033 & 0.083 \\
& B3PW91 & 0.072 & 0.132 & 0.096 & 0.034 & 0.084 \\
& B97-2 & 0.077 & 0.076 & 0.101 & 0.035 & 0.076 \\
& MPW1K & 0.084 & 0.064 & 0.082 & 0.028 & 0.073 \\
HM-GGA & MPW3LYP & 0.089 & 0.068 & 0.116 & 0.039 & 0.084 \\
& BB1K & 0.084 & 0.061 & 0.082 & 0.029 & 0.071 \\
& MPWB1K & 0.086 & 0.059 & 0.075 & 0.029 & 0.071 \\
Average & TPSSh & 0.084 & 0.079 & 0.105 & 0.035 & 0.080 \\
& & 0.087 & 0.083 & 0.102 & 0.041 & 0.084 \\
\hline
\end{tabular}


Table 9. Calculated Mean Unsigned Error (MUE) in the Zn-Ligand Bond-Lengths (Å) by Type of Coordination for the Several Basis Sets Considered in this Study.

\begin{tabular}{lcccccc}
\hline & \multicolumn{3}{c}{ Bonds $(\AA)$} & & Angles $\left({ }^{\circ}\right)$ \\
\cline { 2 - 5 } \cline { 5 - 6 } Basis sets & $\begin{array}{c}\mathrm{Zn}-\mathrm{O} \\
(15 \text { bonds })\end{array}$ & $\begin{array}{c}\mathrm{Zn}-\mathrm{N} \\
(17 \text { bonds })\end{array}$ & $\begin{array}{c}\mathrm{Zn}-\mathrm{S} \\
(8 \text { bonds })\end{array}$ & $\begin{array}{c}\mathrm{Zn}-\mathrm{Cl} \\
(4 \text { bonds })\end{array}$ & $\begin{array}{c}\mathrm{Zn}-\mathrm{All} \\
(44 \text { bonds })\end{array}$ & $\begin{array}{c}\text { Average } \\
(60 \text { angles })\end{array}$ \\
\hline 6-31G(d) & 0.123 & 0.061 & 0.057 & 0.015 & 0.066 & 8.7 \\
$6-311 \mathrm{G}(\mathrm{d}, \mathrm{p})$ & 0.079 & 0.085 & 0.077 & 0.021 & 0.064 & 10.1 \\
CEP-121G & 0.076 & 0.102 & 0.107 & 0.040 & 0.090 & 9.3 \\
LanL2DZ & 0.083 & 0.095 & 0.181 & 0.088 & 0.112 & 10.6 \\
SDD & 0.072 & 0.075 & 0.087 & 0.039 & 0.068 & 9.5 \\
Average & 0.087 & 0.083 & 0.102 & 0.041 & 0.084 & 9.6 \\
\hline
\end{tabular}

For $\mathrm{Zn}-\mathrm{S}$ and $\mathrm{Zn}-\mathrm{Cl}$ bonds, the two all-electron basis sets give considerable better results than the basis sets that use ECPs. Globally, SDD gives the best results for all bond-types, among the three basis sets that use ECPs. LanL2DZ presents the same level of accuracy than CEP-121G in the determination of $\mathrm{Zn}-\mathrm{O}$ bond-lengths, gives slightly better results for $\mathrm{Zn}-\mathrm{N}$ interaction, but is significantly worse for $\mathrm{Zn}-\mathrm{S}$ and $\mathrm{Zn}-\mathrm{Cl}$ bonds, for which CEP-121G presents average MUE values almost 50\% lower.

Table 10 presents the MUE values calculated for each of the 90 combinations of method/basis set, considering all the angles in which Zinc was the central atom. A total of 60 angles present in the 10 Zinc complexes considered were evaluated. The average MUE in the set of 90 combinations tested was of $9.6^{\circ}$.

G96LYP gave the lowest MUE error $\left(7.4^{\circ}\right)$ in the determination of angles among the GGAs and was ranked first in the set of 18 density functionals evaluated. BB95, with an average MUE of $8.5^{\circ}$, gave the best results among the M-GGAs, whereas B97-2 and MPW1K with average MUE of $9.7^{\circ}$ and $9.9^{\circ}$ came first among the H-GGAs. BB1K with an average MUE value of $8.3^{\circ}$ gave the best results among the HM-GGAs. The LSDA density functional SVWN3 gave the worst results in the test for the determination of Ligand-Zn-Ligand angles (average MUE of $12.4^{\circ}$ ). In terms of the basis sets considered, the differences obtained between the several alternatives tested were in general relatively small. Among the all electron basis sets, 6-31G(d) gave an average MUE of $8.7^{\circ}$, whereas $6-311 \mathrm{G}(\mathrm{d}, \mathrm{p})$ resulted in an average MUE of $10.1^{\circ}$. The basis sets that included ECPs showed a good performance in the determination of angles with $\mathrm{Zn}$ as the central atom, with CEP-121G and SDD (average MUEs of $9.3^{\circ}$ and $9.5^{\circ}$, respectively) performing slightly better than LanL2DZ (average MUE of $10.6^{\circ}$ ). Globally, the combinations BB95/CEP-121G and HCTH/CEP-121G were the most successful in the determination of Ligand-Zn-Ligand atoms (average MUEs of $3.1^{\circ}$ and $4.5^{\circ}$ ), whereas the worst results were obtained with SVWN3/CEP-121G, TPSS/CEP-121G and SVWN3/SDD (average MUEs higher than $13.3^{\circ}$ ).

Table 10. Calculated Mean Unsigned Error (MUE) in the Ligand-Zn-Ligand Angles $\left(^{\circ}\right.$ ).

\begin{tabular}{llccccrr}
\hline Type & Functional & $6-31 \mathrm{G}(\mathrm{d})$ & $6-311 \mathrm{G}(\mathrm{d}, \mathrm{p})$ & CEP-121G & LanL2DZ & SDD & Av \\
\hline LSDA & SVWN3 & 9.6 & 11.9 & 15.2 & 12.2 & 13.3 & 12.4 \\
GGA & BLYP & 6.8 & 10.1 & 12.5 & 10.9 & 7.3 & 9.5 \\
& BP86 & 9.2 & 8.8 & 12.2 & 11.0 & 9.3 & 10.1 \\
& G96LYP & 6.8 & 8.1 & 4.7 & 10.7 & 6.8 & 7.4 \\
& HCTH & 6.8 & 9.8 & 4.5 & 10.6 & 6.8 & 7.7 \\
& PBE & 9.3 & 10.3 & 5.3 & 10.1 & 9.3 & 8.9 \\
M-GGA & BB95 & 10.2 & 10.7 & 3.1 & 8.0 & 10.7 & 8.5 \\
& TPSS & 7.0 & 11.3 & 15.1 & 9.8 & 9.6 & 10.6 \\
& VSXC & 10.3 & 11.5 & 4.8 & 12.9 & 13.6 & 10.6 \\
H-GGA & B3LYP & 9.7 & 11.6 & 10.6 & 10.9 & 10.5 & 10.7 \\
& B3P86 & 11.9 & 8.9 & 11.0 & 11.1 & 10.3 & 10.6 \\
& B3PW91 & 6.9 & 10.2 & 13.7 & 10.8 & 9.9 & 10.3 \\
& B97-2 & 8.3 & 10.0 & 9.6 & 10.7 & 9.9 & 9.7 \\
& MPW1K & 8.5 & 9.2 & 10.8 & 11.0 & 9.9 & 9.9 \\
& MPW3LYP & 9.1 & 10.0 & 10.6 & 11.0 & 10.2 & 10.2 \\
HM-GGA & BB1K & 8.4 & 10.9 & 5.7 & 10.8 & 5.8 & 8.3 \\
& MPWB1K & 7.4 & 10.6 & 7.3 & 11.8 & 7.0 & 8.7 \\
& TPSSh & 9.5 & 10.3 & 11.3 & 11.1 & 10.3 & 10.5 \\
Average & & 8.7 & 10.1 & 9.3 & 10.6 & 9.5 & 9.6 \\
& & & & & & & \\
\hline
\end{tabular}


Table 11. Summary of the Main Conclusions Drawn in this Study.

\begin{tabular}{|c|c|}
\hline Density functionals & \\
\hline LSDAs and GGAs & $\begin{array}{l}\text { Significantly worse performance than the other } \\
\text { families of density functionals. }\end{array}$ \\
\hline & $\begin{array}{l}\text { Best Performance with G96LYP, with SVWN3 } \\
\text { giving the worst results }\end{array}$ \\
\hline M-GGAs & $\begin{array}{l}\text { Best performance with TPSS, particularly for } \\
\mathrm{Zn}-\mathrm{O} \text { bonds }\end{array}$ \\
\hline H-GGAs & $\begin{array}{l}\text { Best performance with MPW } 1 \mathrm{~K} \text { and } \mathrm{B} 97-2 \\
\text { B3LYP with only modest performance, but fast }\end{array}$ \\
\hline HM-GGAs & $\begin{array}{l}\text { Best performance with BB1K and MPWB1K } \\
\text { (best in the test) }\end{array}$ \\
\hline Basis sets & \\
\hline All electron & $\begin{array}{l}\text { Performance of } 6-31 \mathrm{G}(\mathrm{d}) \text { and of } 6-311 \mathrm{G}(\mathrm{d}, \mathrm{p}) \\
\text { was basically at the same level in the } \\
\text { determination of bond-lengths and angles } \\
\text { The much higher computational cost of the } \\
6-311 \mathrm{G}(\mathrm{d}, \mathrm{p}) \text { basis set does not justify its use } \\
\text { in geometry optimization of } \mathrm{Zn} \text { complexes }\end{array}$ \\
\hline ECPs & $\begin{array}{l}\text { SDD and CEP- } 121 \mathrm{G} \text { are safe alternatives to } \\
\text { the all-electron basis sets for geometry } \\
\text { optimization }\end{array}$ \\
\hline & $\begin{array}{l}\text { Use of the Los Alamos large-core formulation } \\
\text { is not recommended }\end{array}$ \\
\hline
\end{tabular}

\section{Conclusions}

In this article, we have compared the performance of 18 density functionals and 5 basis sets in the determination of 44 bondlengths and 60 angles involving Zinc in 10 reasonably-sized representative transition metal complexes taken from the Cambridge Structural Database.

The results (summarized in Table 11) show that most combinations density functional/basis set evaluated in this study allow a very reasonable determination of the bond-lengths and angles involving Zn, with a MUE below $0.1 \AA$ for bond-lengths and $10^{\circ}$ for angles. The average MUE values in the full test set were of $0.084 \AA$ and $9.6^{\circ}$, for bond-lengths and angles, respectively. The best performance in the test was obtained with the density functionals MPWB1K and BB1K. These density functionals gave the best average results in the determination of $\mathrm{Zn}$-ligand bond-lengths, and were on the top three best alternatives in bond-length determination for four of the five basis sets tested. The exception was LanL2DZ. In addition, BB1K was also among the three best density functionals in the determination of angles involving $\mathrm{Zn}$. This density functional has also been recently shown in several studies to significantly outperform a large number of commonly available density functionals for the determination of reaction barrier heights. ${ }^{36}$ Our results suggest BB1K and MPWB1K to be very good alternatives for the treatment of Zinc biological complexes. In addition to these two density functionals, a good agreement in the determination of $\mathrm{Zn}$-ligand bond-lengths and angles was also obtained with the H-GGA density functionals MPW1K and B97-2, and with the M-GGA TPSS. This last density functional gave particularly good results in the determination of $\mathrm{Zn}-\mathrm{O}$ bond-lengths. Authors wishing to study $\mathrm{Zn}$ systems with a par- ticularly high prevalence of such bonds should consider the use of this density functional in their calculations.

Globally, the results indicate a significant improvement in accuracy when moving to the more sophisticated density functionals, showing that the technical progress in the field has significant practical results, even in geometry optimization. This difference is particularly relevant when going from the LSDAs to the GGAs and from these to the M-GGAs. In fact, the performance of LSDA and GGA density functionals was significantly worse than that of the remaining density functionals. The only exception was that of the G96LYP density functional, the best among the GGAs tested. The performance of the M-GGAs, H-GGAs, and HM-GGAs evaluated was generically at the same level, varying more among individual density functionals than among the global families of density functionals. The popular B3LYP density functional exhibited a particularly poor performance, giving the highest average MUE for both bond-length and angle determination in the set of density functionals from these three families, although its low computational cost and the relatively small difference in terms of MUE between the several functionals tested, still render this functional a competitive alternative for geometry determination in biological Zinc complexes.

In terms of the basis sets tested, the results show that the use of the more computationally demanding basis sets $6-311 \mathrm{G}(\mathrm{d}, \mathrm{p})$ does not significantly improve the accuracy on the calculated metal-ligand bond-lengths and angles over the values obtained with the more computationally accessible basis set $6-31 \mathrm{G}(\mathrm{d})$. The results also showed that the use of ECPs allows a significant gain in terms of CPU time without a noticeable loss of accuracy in comparison with the two all-electron basis sets tested. The SDD basis set, which uses the Stuttgart-Dresden ECPs, gave particularly good results and was shown to be an excellent alternative to an all-electron description of the more traditional basis sets in the determination of geometrical parameters for Zinc complexes. From our results, the Los Alamos pseudopotencial in its large-core formulation cannot be recommended for the treatment of Zinc complexes. The new and more flexible basis set that employs the Los Alamos ECP - denoted LanL2TZ - also did not succeed in improving the LanL2DZ results for the combinations tested in this study, but the performance of this very promising basis set in other settings should be attempted in the future.

The results presented here show that there are currently several better alternatives to the B3LYP density functional for the determination of metal-ligand bond-lengths and angles in Zinc complexes. BB1K, MPWB1K, MPW1K, B97-2, and TPSS are among the best alternatives to this popular density functional, and are already available in the most popular computational chemistry software packages. A recent benchmarking study evaluated the performance of 38 density functionals in the optimization of geometries for 10 small $\mathrm{Zn}$-model compounds. ${ }^{56}$ In this study, B3LYP was ranked 4th in the test, surpassed by only X3LYP, PW5B95, and M05-2X, density functionals presently not available in most computational chemistry software packages. Other new alternatives are in development and are likely to be made available to the scientific community over the next years. ${ }^{79-86}$ Additional features such as the inclusion of relativistic effects ${ }^{56}$ have also been proved relevant for Zn-ligand inter- 
actions. The reader is advised to take all these aspects into careful consideration before embarking in a new DFT study involving Zinc complexes. More studies, involving different properties and other types of systems, are required to fully demystify the standing of B3LYP, as the first choice for any DFT calculation regardless of the problem at hand, and prepare the field of computational chemistry to take full advantage of the new developments in DFT that have taken place over the last years.

\section{References}

1. Vallee, B. L.; Auld, D. S. Biochemistry 1990, 29, 5647.

2. Vallee, B. L.; Auld, D. S. Proc Natl Acad Sci USA 1990, 87, 220.

3. Lipscomb, W. N.; Strater, N. Chem Rev 1996, 96, 2375.

4. Dudev, T.; Lim, C. Chem Rev 2003, 103, 773.

5. Andreini, C.; Banci, L.; Bertini, I.; Rosato, A. J Proteome Res 2006, 5, 196.

6. Coleman, J. E. Curr Opin Chem Biol 1998, 2, 222.

7. Hahn, S. Nat Struct Mol Biol 2004, 11, 394.

8. Bushnell, D. A.; Westover, K. D.; Davis, R.; Kornberg, R. D. Science 2004, 303, 983.

9. Donaldson, I. M.; Friesen, J. D. J Biol Chem 2000, 275, 13780.

10. Gnatt, A. L.; Fu, J.; Bushnell, D. A.; Kornberg, R. D. Science 2001, 292, 1876

11. Cramer, P.; Bushnell, D. A.; Kornberg, R. D. Science 2001, 292, 1863.

12. Brinckerhoff, C. E.; Matrisian, L. M. Nat Rev Mol Cell Biol 2002, 3, 207.

13. Egeblad, M.; Werb, Z. Nat Rev Cancer 2002, 2, 161.

14. Coussens, L. M.; Fingleton, B.; Matrisian, L. M. Science 2002, 295, 2387.

15. Long, S. B.; Casey, P. J.; Beese, L. S. Nature 2002, 419, 645.

16. Park, H. W.; Boduluri, S. R.; Moomaw, J. F.; Casey, P. J.; Beese, L. S. Science 1997, 275, 1800.

17. Sousa, S. F.; Fernandes, P. A.; Ramos, M. J. J Mol Struct (Theochem) 2005, 729, 125.

18. Sousa, S. F.; Fernandes, P. A.; Ramos, M. J. J Biol Inorg Chem $2005,10,3$.

19. Sousa, S. F.; Fernandes, P. A.; Ramos, M. J. Biophys J 2005, 88, 483.

20. Tobin, D. A.; Pickett, J. S.; Hartman, H. L.; Fierke, C. A.; PennerHahn, J. E. J Am Chem Soc 2003, 125, 9962.

21. Cricco, J. A.; Vila, A. J. Curr Pharm Des 1999, 5, 915.

22. Heinz, U.; Adolph, H. W. Cell Mol Life Sci 2004, 61, 2827.

23. McCall, K. A.; Huang, C. C.; Fierke, C. A. J Nutr 2000, 130, 1437.

24. Penner-Hahn, J. E. Coord Chem Rev 2005, 249, 161.

25. Bertini, I.; Luchinat, C.; Rosi, M.; Sgamellotti, A.; Tarantelli, F. Inorg Chem 1990, 29, 1460.

26. Nakagawa, S.; Umeyama, H. J Theor Biol 1982, 96, 473.

27. Demoulin, D.; Pullman, A. Theor Chim Acta 1978, 49, 161.

28. Liang, J. Y.; Lipscomb, W. N. Biochemistry 1987, 26, 5293.

29. Fermi, E. Rend Accad Naz Lincei 1927, 6, 602.

30. Thomas, L. H. Proc Camb Philos Soc 1927, 23, 542.

31. Hohenberg, P.; Kohn, W. Phys Rev B: Condens Matter 1964, 136, 864.

32. Kohn, W.; Sham, L. J. Phys Rev A: At Mol Opt Phys 1965, 140, 1133.

33. Becke, A. D. Phys Rev A: At Mol Opt Phys 1988, 38, 3098.

34. Becke, A. D. J Chem Phys 1996, 104, 1040.

35. Lee, C.; Yang, W.; Parr, R. G. Phys Rev B: Condens Matter 1988, 37, 785 .
36. Sousa, S. F.; Fernandes, P. A.; Ramos, M. J. J Phys Chem A 2007, 111, 10439.

37. Wang, N. X.; Wilson, A. K. J Chem Phys 2004, 121, 7632.

38. Zhao, Y.; Truhlar, D. G. J Phys Chem A 2004, 108, 6908.

39. Schultz, N. E.; Zhao, Y.; Truhlar, D. G. J Phys Chem A 2005, $109,11127$.

40. Schultz, N. E.; Zhao, Y.; Truhlar, D. G. J Phys Chem A 2005, $109,4388$.

41. Riley, K. E.; Op't Holt, B. T.; Merz, K. M., Jr. J Chem Theory Comput 2007, 3, 407.

42. Riley, K. E.; Brothers, E. N.; Ayers, K. B.; Merz, K. M. J Chem Theory Comput 2005, 1, 546.

43. Zhao, Y.; Pu, J.; Benjamin, J. L.; Truhlar, D. G. Phys Chem Chem Phys 2004, 6, 673.

44. Zhao, Y.; Truhlar, D. G. J Phys Chem A 2005, 109, 5656.

45. Zhao, Y.; Gonzalez-Garcia, N.; Truhlar, D. G. J Phys Chem A 2005, 109, 2012.

46. Zhao, Y.; Truhlar, D. G. J Phys Chem A 2006, 124, 224105.

47. Brothers, E. N.; Merz, K. M. J Phys Chem A 2004, 108, 2904.

48. Curtiss, L. A.; Redfern, P. C.; Raghavachari, K. J Chem Phys 2005, 123, 124107.

49. Zhao, Y.; Tishchenko, O.; Truhlar, D. G. J Phys Chem B 2005, 109, 19046.

50. Zhao, Y.; Truhlar, D. G. J Chem Theory Comput 2005, 1, 415.

51. Zhao, Y.; Truhlar, D. G. J Phys Chem A 2006, 110, 5121.

52. Zhao, Y.; Truhlar, D. G. Phys Chem Chem Phys 2005, 7, 2701.

53. Waller, M. P.; Buhl, M. J Comput Chem 2007, 28, 1531.

54. Buhl, M.; Kabrede, H. J Chem Theory Comput 2006, 2, 1282.

55. Buhl, M.; Reimann, C.; Pantazis, D. A.; Bredow, T.; Neese, F. J Chem Theory Comput 2008, 4, 1449.

56. Amin, E. A.; Truhlar, D. G. J Chem Theory Comput 2008, 4, 75.

57. Himo, F. Theor Chem Acc 2006, 116, 232.

58. Leopoldini, M.; Marino, T.; Michelini, M. D.; Rivalta, I.; Russo, N.; Sicilia, E.; Toscano, M. Theor Chem Acc 2007, 117, 765.

59. Ramos, M. J.; Fernandes, P. A. Acc Chem Res 2008, 41, 689.

60. Liao, R. Z.; Yu, J. G.; Raushel, F. M.; Himo, F. Chemistry 2008, $14,4287$.

61. Leopoldini, M.; Chiodo, S. G.; Toscano, M.; Russo, N. Chemistry 2008, 14, 8674.

62. Kim, J.; Tsai, P. C.; Chen, S. L.; Himo, F.; Almo, S. C.; Raushel, F. M. Biochemistry 2008, 47, 9497.

63. Leopoldini, M.; Marino, T.; Russo, N.; Toscano, M. Int J Quantum Chem 2008, 108, 2023.

64. Siegbahn, P. E. M.; Tye, J. W.; Hall, M. B. Chem Rev 2007, 107, 4414.

65. Sousa, S. F.; Fernandes, P. A.; Ramos, M. J. Proteins 2007, 66, 205.

66. Hopmann, K. H.; Himo, F. Eur J Inorg Chem 2008, 22, 3452.

67. Picot, D.; Ohanessian, G.; Frison, G. Inorg Chem 2008, 47, 8167.

68. Tye, J. W.; Darensbourg, M. Y.; Hall, M. B. Inorg Chem 2008, 47, 2380.

69. Estiu, G. L.; Rasia, R. M.; Cricco, J. A.; Vila, A. J.; Zerner, M. C. Int J Quantum Chem 2002, 88, 118.

70. Dudev, T.; Lim, C. Ann Rev Biophys 2008, 37, 97.

71. Holt, B. T. O.; Merz, K. M. Biochemistry 2007, 46, 8816.

72. Sousa, S. F.; Fernandes, P. A.; Ramos, M. J. Theor Chem Acc 2007, 117, 171.

73. Estiu, G.; Merz, K. M. Biochemistry 2006, 45, 4429.

74. Sousa, S. F.; Fernandes, P. A.; Ramos, M. J. J Phys Chem B 2007, $111,9146$.

75. Allen, F. H. Acta Crystallogr Sect B 2002, 58, 380.

76. Tamames, B.; Sousa, S. F.; Tamames, J.; Fernandes, P. A.; Ramos, M. J. Proteins 2007, 69, 466. 
77. Lee, Y. M.; Lim, C. J Mol Biol 2008, 379, 545.

78. Patel, K.; Kumar, A.; Durani, S. Biochim Biophys Acta 2007, 1774, 1247.

79. Zhao, Y.; Truhlar, D. G. Theor Chem Acc 2008, 120, 215.

80. Zhao, Y.; Truhlar, D. G. J Chem Phys 2006, 125, 194101.

81. Zhao, Y.; Schultz, N. E.; Truhlar, D. G. J Chem Theory Comput 2006, 2, 364.

82. Schwabe, T.; Grimme, S. Phys Chem Chem Phys 2007, 9, 3397.

83. Antony, J.; Grimme, S. Phys Chem Chem Phys 2006, 8, 5287.

84. Grimme, S. J Comput Chem 2006, 27, 1787.

85. Schwabe, T.; Grimme, S. Phys Chem Chem Phys 2006, 8, 4398.

86. Zhao, Y.; Truhlar, D. G. Acc Chem Res 2008, 41, 157.

87. Frisch, M. J.; Trucks, G. W.; Schlegel, H. B.; Scuseria, G. E.; Robb, M. A.; Cheeseman, J. R.; Montgomery, J. A.; Vreven, T.; Kudin, K. N.; Burant, J. C.; Millam, J. M.; Iyengar, S. S.; Tomasi, J.; Barone, V.; Mennucci, B.; Cossi, M.; Scalmani, G.; Rega, N.; Petersson, G. A.; Nakatsuji, H.; Hada, M.; Ehara, M.; Toyota, K.; Fukuda, R.; Hasegawa, J.; Ishida, M.; Nakajima, T.; Honda, Y.; Kitao, O.; Nakai, H.; Klene, M.; Li, X.; Knox, J. E.; Hratchin, H. P.; Cross, J. B.; Adamo, C.; Jaramillo, J.; Gomperts, R.; Stratmann, R. E.; Yazyev, O.; Austin, A. J.; Cammi, R.; Pomelli, C.; Ochterski, J. W.; Ayala, P. Y.; Morokuma, K.; Voth, G. A.; Salvador, P.; Dannenberg, J. J.; Zakrzewski, V. G.; Dapprich, S.; Daniels, A. D.; Strain, M. C.; Farkas, O.; Malik, D. K.; Rabuck, A. D.; Raghavachari, K.; Foresman, J. B.; Ortiz, J. V.; Cui, Q.; Baboul, A. G.; Clifford, S.; Cioslowski, J.; Stefanov, B. B.; Liu, G.; Liashenko, A.; Piskorz, P.; Komaromi, I.; Martin, R. L.; Fox, D. J.; Keith, T.; Al-Lahan, A.; Peng, C. Y.; Nanayakkara, A.; Challacombe, M.; Gill, P. M. W.; Johnson, B.; Chen, W.; Wong, M. W.; Gonzalez, C.; Pople, J. A. Gaussian 03 2004, Revision C. 02; Gaussian, Inc.: Wallingford, CT, 2004.

88. Perdew, J. P. Phys Rev B: Condens Matter 1986, 33, 8822.

89. Perdew, J. P. Electronic Structure of Solids '91; Akademie Verlag: Berlin, Germany, 1991.

90. Hamprecht, F. A.; Cohen, A. J.; Tozer, D. J.; Handy, N. C. J Chem Phys 1998, 109, 6264.

91. Zhao, Y.; Lynch, B. J.; Truhlar, D. G. J Phys Chem A 2004, 108, 2715.

92. Gill, P. M. W. Mol Phys 1996, 89, 433.

93. Adamo, C.; Barone, V. J Chem Phys 1998, 108, 664.

94. Lynch, B. J.; Fast, P. L.; Harris, M.; Truhlar, D. G. J Phys Chem A $2000,104,4811$.

95. Zhao, Y.; Truhlar, D. G. J Phys Chem A 2004, 108, 6908.
96. Perdew, J. P.; Burke, K.; Ernzerhof, M. Phys Rev Lett 1996, 77, 3865.

97. Vosko, S. H.; Wilk, L.; Nusair, M. Can J Phys 1980, 58, 1200.

98. Slater, J. C. The Self-Consistent Field for Molecular and Solids; McGraw-Hill: New York, USA, 1974.

99. Tao, J.; Perdew, J. P.; Staroverov, V. N.; Scuseria, G. E. Phys Rev Lett 2003, 91, 146401.

100. Van Voorhis, T.; Scuseria, G. E. J Chem Phys 1998, 109, 400.

101. Lynch, B. J.; Zhao, Y.; Truhlar, D. G. J Phys Chem A 2003, 107, 1384.

102. Cundari, T. R.; Stevens, W. J. J Chem Phys 1993, 98, 5555.

103. Stevens, W. J.; Krauss, M.; Basch, H.; Jasien, P. G. Can J Chem 1992, 70, 612.

104. Stevens, W. J.; Basch, H.; Krauss, M. J Chem Phys 1984, 81, 6026.

105. Hay, P. J.; Wadt, W. R. J Chem Phys 1985, 82, 299.

106. Wadt, W. R.; Hay, P. J. J Chem Phys 1985, 82, 284.

107. Hay, P. J.; Wadt, W. R. J Chem Phys 1985, 82, 270.

108. Andrae, D.; Haussermann, U.; Dolg, M.; Stoll, H.; Preuss, H. Theor Chim Acta 1990, 77, 123.

109. Dolg, M.; Wedig, U.; Stoll, H.; Preuss, H. J Chem Phys 1987, 86, 866.

110. Petrusenko, S. R.; Kokozei, V. N. Zh Neorg Khim 1996, 41, 1294.

111. Belsky, V. K.; Streltsova, N. R.; Bulychev, B. M.; Storozhenko, P. A.; Ivankina, L. V.; Gorbunov, A. I. Inorganica Chim Acta 1989, 164,211

112. Cameron, E. M.; Louch, W. E.; Cameron, T. S.; Knop, O. Z Anorg Allg Chem 1998, 624, 1629.

113. Hill, M. R.; Jones, A. W.; Russell, J. J.; Roberts, N. K.; Lamb, R. N. Inorganica Chim Acta 2005, 358, 201.

114. Bertels, J.; Mattes, R. Z Naturforsch B Chem Sci 1985, 40, 1068.

115. Johansson, A.; Hakansson, M. Acta Crystallogr Sect E 2004, 60, M955.

116. Xiang, J.; Lin, X. C. Acta Crystallogr Sect E 2006, 62, M593.

117. Bond, A. D.; Jones, W. J Chem Soc Dalton Trans 2001, 20, 3045.

118. Buntine, M. A.; Hall, V. J.; Kosovel, F. J.; Tiekink, E. R. T. J Phys Chem A 1998, 102, 2472.

119. Martin, A.; Orpen, A. G. J Am Chem Soc 1996, 118, 1464.

120. Orpen, A. G. Acta Crystallogr Sect B 2002, 58, 398.

121. Orpen, A. G.; Quayle, M. J. J Chem Soc Dalton Trans 2001, 10, 1601.

122. Roy, L. E.; Hay, P. J.; Martin, R. L. J Chem Theory Comput 2008, 4, 1029. 